\title{
In-situ TEM Coupled with AFM Cantilever for Direct Observation of Li Dendrite Nucleation and Growth Under Stress
}

\author{
Yang $\mathrm{He}, \mathrm{Wu} \mathrm{Xu}$ and Chongmin Wang \\ Pacific Northwest National Laboratory, Richland, Washington, United States
}

Lithium (Li) metal has very low standard electrochemical redox potential and very high theoretical specific capacity, making it an ultimate anode material for rechargeable batteries. However, its application has been impeded by the formation of Li whiskers, which not only considerably consumes electrolyte and active Li, but also may lead to short-circuit of the battery. Tackling of these obstacles is limited by the elusive understanding on the intrinsic formation mechanisms of the Li whiskers and their growth behavior under the constraints from the separator.

By coupling an atomic force microscopy (AFM) cantilever into a solid-cell setup in an environmental transmission electron microscopy (ETEM), we directly captured the Li whisker nucleation and growth processes under elastic constraint that mimics the effect of a separator. It is found that the growth mode and morphology of deposited Li can be critically related to the ionic conductivity of the initial solid electrolyte layer (SEI) that is introduced by the basic reactions of freshly deposited Li metal with the surrounding gas molecules. The finding is further validated in batteries using practical electrolyte systems. Moreover, Li whisker growing under stress (e.g. from the separator) could yield, buckle, kink, or stop axial growth [1].

Commercially available AFM cantilevers (from Bruker AFM Probes) were used in this investigation. The cantilever tip was cut into a flat surface by using a Focused Ion Beam (Helios, FEI, USA). A layer of Ni of $\sim 15 \mathrm{~nm}$ in thickness was coated on the cantilever by using an ion beam sputter to make the AFM cantilever conductive; since pure $\mathrm{Ni}$ does not react with $\mathrm{Li}$, it serves as a current collector for the nanoscale solid state battery. The Ni-coated AFM chip was pasted on a platinum rod by using silver glue and then loaded on to the fixed end of a Nanofactory-STM holder. The spring constant of the AFM cantilever was selected to mimic the stiffness of a separator used in a real lithium ion battery. The AFM cantilevers with spring constants of $0.07,0.1,0.4,3 \mathrm{nN} \cdot \mathrm{nm}^{-1}$ were used in our study, which can reasonably mimic the elastic interaction between the separator and the Li whisker growth.

Pure bulk Li metal was scratched onto a tungsten rod and then fixed to the piezo-controlled end of a Nanofactory-STM holder. The holder was then inserted into a Titan 80-300 Environmental TEM which operated at $300 \mathrm{kV}$, equipped with an imaging lens spherical aberration corrector and a charge-coupled device to record the electrochemical process at 2 frames per second). The oxidation layer formed on the Li metal surface due to a few seconds exposure to air (which consists mainly of $\mathrm{Li}_{2} \mathrm{O}$, and possibly trivial amount of hydroxide, nitride/amide, and carbonate) and acted as the solid electrolyte. Note that the experimental setup was stabilized in the TEM for $\sim 1 \mathrm{~h}$ before the tests, to minimize the drift of the interested region and increase the accuracy of the AFM-deflection (i.e. force) measurement. The $\mathrm{CO}_{2} \mathrm{gas}$ was leaked into the TEM chamber at a controlled pressure of $10^{-2} \sim 10^{-3}$ mbar by using a lab-developed auxiliary gas-delivery system. The Li metal was manipulated by the piezo-system on the holder to contact the AFM cantilever that was fixed on the other end of the Nanofactory-STM holder. Then, a constant bias of $2 \mathrm{~V}$ was applied between the two electrodes: Ni on AFM as the working-electrode; Li metal as counterelectrode to drive the electrochemical reactions. Under a constant driving force for deposition, the Li metal 
was electrochemically oxidized to $\mathrm{Li}^{+}$ions which migrated across the solid electrolyte, reduced to $\mathrm{Li}$ metal, and hence deposited on the Ni film that was coated on the AFM cantilever tip. The deposition process was recorded by the charge coupled device and the deposited Li was characterized by using electron diffraction.

We directly captured the nucleation and growth behavior of Li whiskers under elastic constraint that mimics the effect of a separator. We show that Li deposition is initiated by a sluggish nucleation of a single crystalline Li particle with no preferential growth directions. We discovered that carbonate species in the initial solid electrolyte interphase that is very adjacent to the developing Li metal plays a decisive role in the subsequent formation of $\mathrm{Li}$ with a whisker morphology. Characteristically, the growing $\mathrm{Li}$ whisker, depending on both intrinsic and extrinsic conditions, can yield, buckle, kink, or stop (axial) growth under elastic constraint from the separator. Based on our force measurements, the growth force generated by the growing whiskers are in the range of $0.1 \sim 10 \mu \mathrm{N}$. These findings reveal the intrinsic cause and give a clear process on the formation and behavior of dendritic Li under stress, providing the muchneeded insights for solving the $\mathrm{Li}$ whisker formation from the root cause rather than as currently containing it, and therefore potentially leading to the safe operation of Li metal anode in batteries [2].

\section{References}

[1] Yang He, Xiaodi Ren, Yaobin Xu, Mark Engelhard, Xiaolin Li, Jie Xiao, Jun Liu, Ji-Guang Zhang, $\mathrm{Wu} \mathrm{Xu}$, and Chongmin Wang, "Origin of Lithium Whisker Formation and Growth under Stress", Nature Nanotechnology, 2019, 14, 1042-1047.

[2] The work was conducted in the William R. Wiley Environmental Molecular Sciences Laboratory (EMSL), a national scientific user facility sponsored by DOE's Office of Biological and Environmental Research and located at PNNL. 